\title{
Milliliter per Day per Milligram per Day
}

National Cancer Institute

\section{Source}

National Cancer Institute. Milliliter per Day per Milligram per Day. NCI Thesaurus. Code C120762.

A unit of concentration equal to milliliter per day divided by milligram per day. 